\title{
LOS TERRITORIOS CAMPESINOS Y AGROALIMENTARIOS, SON LA FIGURA TERRITORIAL CAMPESINA LEGITIMA QUE TIENE UNA DOBLE LUCHA PARA SER REALIDAD
}

\author{
The Agri-Food Peasant Territories are the legitimate peasant \\ territorial figure that has a double fight to become reality
}

\author{
Wilmar Harley Castillo-Amorocho*
}

Recibido:27 de abril. 2020. Aceptado: 27 de junio de 2020. Publicado: 01 de julio 2020.

Forma de citar este artículo en APA:

Castillo-Amorocho, W. H., (2020, julio-diciembre). Los Territorios Campesinos Agroalimentarios, son la figura territorial campesina legítima que tienen una doble lucha para ser realidad. Revista CoPaLa, Construyendo Paz Latinoamericana 10 (año 5), pp. 69-82. DOI:10.35600.25008870.2020.10.0166, Recuperado desde: http://revistacopala.net/index.php/ojs/article/view/4

\section{Resumen}

El presente artículo profundiza en los retos que tiene la figura territorial campesina Territorio Campesino Agroalimentario del macizo colombiano ubicado en el norte del departamento de Nariño y sur del departamento del Cauca, expuestos en la tesis de maestría de Nicolás Muñoz, la cual fue analizada a la luz de la dinámica del conflicto armado, la normatividad en materia de territorios del actual gobierno nacional junto a ejemplos que ocurren en otros territorios del país, como el Catatumbo. El escenario para lograr solucionar los retos no es favorable tanto a nivel nacional y local, pero la historia de la organización campesina que impulsa el TECA y el arraigo en el territorio ha permitido sostenerse en el macizo como alternativa de vida para las comunidades.

\section{Palabras clave}

Territorio Campesino Agroalimentario, Plan de Vida Comunitario, Retos, organización campesina.

Comunicador social-periodista de la Universidad del Tolima, con experiencia en radio comunitaria y medios alternativos. Columnista de el medio virtual Contra Punto, de El Salvador. Actualmente community manager de la Asociación Nacional Campesina Coordinador Nacional Agrario, articulada al movimiento social y político Congreso de los Pueblos. whcastilloa@gmail.com 


\section{Abstract}

This article delves into the challenges of the peasant land figure Agri-Food Farmers Territory of the Colombian massif located in the north of the department of Nariño and south of the department of Cauca, exhibited in the master's thesis of Nicolás Muñoz, which was analyzed in the light of the dynamics of the armed conflict, the regulations regarding territories of the current national government along with examples that occur in other territories of the country , like the Catatumbo. The scenario for solving the challenges is not favorable both at the national and local level, but the history of the peasant organization that drives TECA and the roots in the territory has allowed it to be sustained in the massif as an alternative of life for the communities.

\section{Keywords}

Agri-Food Farmers Territory, Community Living Plan, Challenges, peasant organization. 
En Colombia, existen diferentes organizaciones campesinas de carácter nacional y regional cuyos puntos en común son entre otros, la Reforma Agraria, la soberanía alimentaria, fortalecimiento de la economía propia; sin dejar a un lado diferencias en la concepción organizativa o de movilización. Entre ellas existe el Coordinador Nacional Agrario, con 23 años de existencia actualmente la conforma 60 procesos agrarios distribuidos en 22 departamentos.

Además de incluir en su proyecto de nación los puntos mencionados arriba, también tienen una línea estratégica de constituir figuras territoriales campesinas con el objetivo de tener autonomía y soberanía en las decisiones que tomen en esa franja territorial delimitada por la comunidad. Esta apuesta se denomina Territorio Campesino Agroalimentario (TECA) y para comprenderlo se debe explicar también qué son los Planes de Vida Comunitarios (PVC).

Así que los PVC no son más que el ejercicio de planeación, de proyección colectiva del futuro deseado desde la cotidianidad de las personas, familias y comunidades en un territorio especifico, de manera sistemática y organizada que permita ordenar ideas, estrategias y acciones colectivas para lograr ese futuro deseado (2015). Cada pueblo (campesinado, indígena, afrodescendiente) con sus condiciones históricas, con la influencia de sus circunstancias específicas de vida, el tipo de relación con el territorio, las formas de intercambio económico y cultural, el diálogo con las instituciones incide en como participarán los miembros de la comunidad concertadamente y sin romper el tejido social (2015).

Ahora, la postura de clase del CNA agrega por qué los PVC son una alternativa al desarrollo moderno: "[ [... históricamente han estado en función de los que más tienen y orientados al crecimiento económico excluyente a costa de nuestros bienes comunes, nuestros derechos y la vida misma; desconociendo las demandas de vida digna del campesinado y el pueblo colombiano y amenazando los territorios con sus megaproyectos extractivistas y de agronegocio.” (p. 13, 2015).

La relación con los TECA subyace en materializar esta concepción de la vida comunitaria en un tipo de orden territorial consecuente con esta, por lo que: “Es un territorio concebido (pensado), habitado y organizado por nuestras familias, comunidades y organizaciones campesinas orientado por un Plan de Vida digna Campesino, resultado de procesos organizativos, sociales, políticos, económicos y culturales. Allí, como campesinos 
hombres y mujeres construimos nuestras relaciones sociales y comunitarias y tenemos una relación directa y especial con la tierra, la naturaleza y el agua, fruto de procesos y prácticas sociales y productivas donde se unen el pasado y el presente. En los Territorios Agroalimentarios desarrollamos actividades como la producción agrícola, pecuaria, forestal, pesquera, así como pequeña minería combinada con agricultura, en las que los productos generados como el Maicito, el Platanito, la Papita, la Yuquita, el Pescado nos sirven para nuestra propia alimentación, pero también los comercializamos en los mercados de nuestros pueblos o plazas de las grandes ciudades para que las comunidades de la ciudad también se alimenten.” (CNA, p. 7, 2015).

Cabe resaltar que los TECA no son un paso obligatorio dentro de una receta que se reproduce descontextualizadamente, existen territorios donde se impulsa el PVC pero sin el TECA, debido a las condiciones de la organización campesina, las circunstancias socio-económicas, por la agudización del conflicto armado. Ahora bien, donde se han constituido los TECAS es debido al avance organizativo de la comunidad y la organización campesina.

\section{El Territorio Campesino Agroambiental de Vida, Agua y Dignidad del norte de Nariño y sur del}

\section{Cauca}

Este punto se desarrollará a partir de la investigación hecha por Nicolás Muñoz (2017) que se caminó este territorio y conversó con sus protagonistas, se abordará a partir de los retos y perspectivas que el autor concluyó en su investigación (Muñoz, p.112, 2017) y a partir de ahí reflexionar sobre la posible consecución o no de los retos abordados.

No sin antes hacer el recorrido del Macizo colombiano y posteriormente de este TECA para ubicar mejor la reflexión. Esta macro región se caracteriza por ser fuente de agua dulce para el 70\% de la población colombiana. Allí nacen 65 cuerpos lagunares de donde nacen los ríos Magdalena, Cauca (estos dos atraviesan el país y desembocan en el océano Atlántico, al norte del país), Caquetá, Patía, Putumayo y Saldaña. El macizo se localiza en la zona alta andina donde la Cordillera central se divide en las cordilleras central y oriental, con una extensión de 4,356.228,87 ha integrado parte de los departamentos de Cauca, Nariño, Putumayo, Caquetá, Huila, Tolima y Valle del Cauca. Al norte limita con el rio Quindío, al oriente con el municipio de Rovira, Tolima; al occidente con el rio Juanambú (municipio de Taminango, Nariño) y al sur con la parte inferior del municipio de Mocoa, del departamento de Putumayo (Muñoz, 2017). (ver mapa no. 1)

La economía de este territorio recae en la agricultura de pequeña y mediana propiedad, donde se destacan los cultivos de maíz en el clima templado y frio; en clima de Páramo la papa y en el cálido la caña de azúcar, el 
plátano, la yuca y el maní junto a la ganadería en menor escala, distribuidos en los 81 municipios que constituyen esta macro región del suroccidente colombiano. Así mismo, la población se divide en resguardos indígenas, comunidades indígenas civiles (Yanaconas)y propiedad campesina distribuidas en dos formas: el modo de vida urbano y modo de vida rural; en la primera su población es mayormente mestiza y afrocolombiana que a diferencia del área rural viven básicamente comunidades indígenas y campesinas (Muñoz, 2017).

En el caso del TECA del Macizo lo constituyen 16 municipios (catorce de Nariño y dos del Cauca) Arboleda, Belén, Buesaco, Chachaguí, Colón, La Cruz, San Bernardo, San Lorenzo, San José de Albán, San Pablo, San Pedro de Cartago, Taminango, Tablón de Gómez; del Cauca son Florencia, Mercaderes y algunos corregimientos de Bolívar. En la economía de esta parte de la macro región, se destaca la producción de café (66.63\% de los predios) en áreas de minifundio. Dos sub-cuencas del Río Patía: la cuenca del Río Juanambú y la cuenca del Río Mayo surten los riegos para este cultivo. En ese sentido, el mayor consumo de agua es utilizado para el sector agrícola (45.6\%), seguido por el consumo humano y uso doméstico; en menor medida por el sector servicios y el sector pecuario (Muñoz, 2017).

La cuenca del río Juanambú atraviesa los municipios del TECA: Albán, Arboleda, Buesaco, Tablón de Gómez, San Bernardo, San Lorenzo, San Pedro de Cartago y Taminango. La cuenca del río Mayo y comprende los siguientes municipios del TECA: La Cruz, La Unión, San Lorenzo, San Pablo, Colón, Taminango, Belén, San Pedro de Cartago, Mercaderes y Florencia (Cauca). Como el 80\% de los ingresos provienen de actividades agropecuarias, se presentan bajos procesos de transformación industrial e informalidad comercial, la industria manufacturera solamente se presenta en el municipio de Belén con la industria del cuero (Muñoz, 2017).

Los y las protagonistas de esta apuesta territorial son el Comité de Integración del Macizo Colombiano -CIMAcomo proceso territorial que ha buscado la construcción de alternativas de institucionalidad propias bajo un criterio de autonomía e independencia (2017). Por ello, su historia se relata en diferentes etapas. La primera inicia con movilizaciones a partir de 1985 en los municipios de Bolívar y luego Sucre en 1986; Santa Rosa y Almaguer (1987); nuevamente Bolívar en 1988 y Almaguer en 1990. Para 1991, nace el CIMA por medio de la Asamblea de delegados con una agenda de cuatro puntos: Impulsar la integración regional, realizar el primer encuentro cultural del Macizo, sacar el periódico regional y organizar el Primer paro Cívico regional de los municipios del Macizo (Muños, p. 15, 2017).

La segunda etapa es de 1991 a 1996, donde se produce la movilización concertada entre el Macizo y el sur del Cauca que dio como resultado la negociación directa con el gobierno nacional y se aborda la propuesta de 
desarrollo para el macizo como región, conocido como Plan de Vida, Agua y Dignidad del Macizo (Muños, p. 16, 2017). El tercer periodo empieza con las asambleas de líderes del Macizo en 1997, luego se realizaron las tres cumbres del Macizo colombiano al siguiente año (1998) para finalizar este periodo con el Primer Paro Cívico del Suroccidente Colombiano en noviembre de 1999 (Muños, p. 16, 2017).

De aquí en adelante, se continúa el trabajo territorial del CIMA en medio de los dos gobiernos de Álvaro Uribe que trajo consigo un debilitamiento preponderante no solo en el movimiento agrario sino en el movimiento social y político en su conjunto, debido a sus políticas que acrecentaron la entrada de multinacionales, el neoliberalismo y el factor paramilitar también generó ese debilitamiento, pues este grupo armado para-estatal no solo confrontó a las guerrillas sino que persiguió al movimiento social y político.

Continuando con la trayectoria del CIMA en el suroccidente colombiano, la propuesta del TECA es resultado de la historia organizativa, de movilización y cultura campesina que inició en los 80’s materializando los principios de autonomía, independencia y arraigo del campesinado macizeño (2017).

\section{EI TECA: la gente decide qué hacer y cómo vivir}

En el siglo XX colombiano, la guerra civil fue protagonizada por amplias franjas del pueblo, que acababan su vida bajo el auspicio de las dirigencias del Partido Liberal y Conservador, debido a esto se produjo un fenómeno de autonomía y sobrevivencia campesina señalado como "Repúblicas independientes" por el congresista conservador Álvaro Gómez generando un debate y controversia nacional y su vez desembocando en ataques militares del Estado contra estas zonas. (Navarro, 2014).

Hoy en día, el control estatal no ha cambiado su forma de operar con sus instituciones, principalmente la militar (encaminada a la guerra contra la insurgencia) y de la mano con la institucionalidad judicial (Fiscalía principalmente) para judicializar no solo a la insurgencia sino también a las comunidades que se contrapongan a los megaproyectos extractivos ${ }^{1}$. Esto claro bajo el carácter totalizador del Estado moderno que cobija a las comunidades bajo una sola forma de entender la nación. (Dussel, 2001)

En el mismo sentido, Santos (2000) nos recuerda la construcción del espacio social por medio de las relaciones sociales de sus habitantes, dentro de una Totalidad implicando una combinación de la carga simbólica y material con la cultura hegemónica de la Totalidad. En la construcción de los TECAS se configuran las

\footnotetext{
${ }^{1}$ En este medio de comunicación se puede conocer el seguimiento a los montajes judiciales contra la comunidad campesina por parte de la Fiscalía y la ECOPETROL y la multinacional Frontera Energy y el Ministerio de Defensa, gracias a contratos económicos realizados entre las empresas y las instituciones. https://trochandosinfronteras.info/caso-san-luis-de-palenque-fiscalia-fuerza-publica-y-empresas-una-alianza-criminal/
} 
relaciones sociales con una carga contra-hegemónica (Santos, 2000) que en su proceso de consecución va instalando otra totalidad, desde los intereses y valores de la clase social campesina.

Así lo reafirma un líder campesino del TECA del Macizo al explicar el tipo de relación que despliegan las comunidades al interior del macizo y del TECA:

"Reconocer como habitantes de este ecosistema, vivir en armonía con la tierra, el territorio, es tener en cuenta de manera consciente, los cíclos del agua, la biodiversidad, las semillas que son la base de la alimentación, la fauna, la flora [...] hacer conciencia de donde estamos parados, que estamos haciendo como comunidad para conservar los elementos vitales" (Daza, 2020, entrevista).

Otro ejemplo del desprendimiento con el Estado moderno está en las comunidades campesinas e indígenas del territorio del Catatumbo ${ }^{2}$, que no han constituido el TECA, pero todo su ser y actuar están basados en el PVC:

"El Plan de Vida va mucho más allá de un plan de alimentación, el Plan de Vida es nuestro pensamiento y nuestra postura política frente al ambiente, frente al territorio, frente a las relaciones que se construyen entre comunidades, frente a la posición que hay que tomar en las decisiones políticas que se han venido construyendo en el día a día para la permanencia; también es el Plan de Vida el que nos lleva a pensar cómo construir y en hacer ese nuevo gobierno desde las comunidades desde una apuesta del Poder Popular" (Cifuentes, 2018, p.61).

La noción de poder que apropian los sujetos de los TECAS, nace del sujeto popular o pueblo, como categoría que aglutina la contra-hegemonía desde los otros sectores sociales diferentes a la clase obrera, cuyas culturas, ideologías, prácticas históricas e intereses se contra ponen a la clase que reproduce el capitalismo (Dussel, 2001). De la misma manera, la dignidad también se mueve entre la telaraña del tejido social que nace en propuesta del TECA. Entendiéndola desde el aporte de Dussel, al referirse a esta como el valor fundante (negado) de todos los valores del ser humano:

"En todo tipo de «sistema» (sea político, económico, pedagógico, de género, de raza, etc.) los miembros que ejercen la hegemonía del mismo excluyen, frecuentemente no intencionalmente (unintentional diría Adam Smith), a sujetos éticos, que sufrirán, al comienzo sin conciencia alguna, dicha dominación como una «negación» a su dignidad.” (p. 140, 2007)

${ }^{2}$ El Catatumbo, se ubica en el departamento de Norte de Santander, limita al norte y occidente con la República Bolivariana de Venezuela, por el oriente con el sur del departamento de Cesar y al sur limita con los municipios de Cúcuta, Puerto Santander, El Zulia y Villa del Rosario. Está compuesto por 11 municipios: Ábrego, Convención, El Carmen, El Tarra, Hacarí, La Playa, Ocaña, San Calixto, Sardinata, Teorama y Tibú. Recibe su nombre por el río Catatumbo. 
Al no tener un reconocimiento en la Constitución de 1991 de sujeto campesino, como pasa al contrario con el pueblo indígena y afrodescendiente, este sector exige su reconocimiento político y legal. La construcción de los TECAS, trae consigo la lucha del reconocimiento del campesinado como sujeto político de derechos, es decir, la lucha por rescatar su dignidad y que los otros sectores, incluyendo el Estado, lo reconozca también.

Otro ejemplo de la lucha por el rescate y reconocimiento de la dignidad campesina, por medio de figuras territoriales, son las Zonas de Reserva Campesina ${ }^{3}$ (ZRC) que, si cuentan con la protección legal de la ley 160 de 1994, del Decreto 1777 de 1996 y del Acuerdo de 1996, pero los sucesivos gobiernos no han cumplido su cumplimiento desde los 90’s.

Entre los objetivos comunes de las ZRC son la superación de las causas de conflictos sociales y ambientales, el fomento y estabilización de la economía campesina, el mejorar la calidad de la vida de los pobladores rurales, el establecimiento de un marco de armonización de los diversos intereses sobre la tierra, a partir de la definición de políticas dentro de un ordenamiento social, productivo y ambiental participativo, para acceder a tierra, bienes públicos, programas y proyectos diseñados en un enfoque de derechos y sostenibilidad (FAO, 2018).

\section{¿Cuáles son los retos del TECA del Macizo?}

La investigación de Muñoz permite no solo conocer el alcance político y territorial de la propuesta del movimiento campesino del macizo, también deja conocer los retos que tiene este campesinado organizado para seguir consolidando el TECA:

"Necesidades Básicas Insatisfechas. El TCA debe convertirse en una herramienta para el mejoramiento de la calidad de vida y bienestar de la población campesina. El reto que se avizora no es menor, ya que las necesidades básicas insatisfechas (NBI) en los municipios del norte de Nariño aumentan visiblemente del casco urbano al área rural de los municipios, que es donde se desenvuelve la mayor parte de la vida campesina.

En los municipios de la cuenca del río Mayo por ejemplo, existe un promedio de 23,93\% de NBI en las cabeceras municipales, mientras que, en el resto, las NBI se incrementan el doble con un promedio de $48,72 \%$.

La situación de los municipios de la cuenca del Juanambú no es más alentadora. El promedio de NBI es superior al del departamento con un porcentaje del 51,79\%, mientras el promedio del departamento es de 43,79\%. Excluyendo del cálculo a los municipios de la cuenca que no pertenecen al TCA, la diferencia es todavía más

${ }^{3}$ Esta figura territorial campesina es impulsada por la Asociación Nacional de Zonas de Reserva Campesina (ANZORC). 
alarmante, porque el promedio de NBI para la zona rural es de 61,34\%, casi el doble del promedio para las cabeceras que es del 31.29\%. (Muñoz, p. 113, 2017)

El siguiente reto expuesto por el autor es la protección de los recursos ambientales:

"El medio físico del Macizo está conformado por un complejo geo hidrológico que contiene 65 cuerpos lagunares en la Alta Montaña, 13 páramos y ecosistemas de flora y fauna. Las áreas de páramo del Macizo equivalen a 3.036,92 km2, que representan el $21 \%$ del total de áreas del páramo del país y se encuentran ubicadas en el Parque Nacional Natural (PNN) Las Hermosas, PNN Nevado del Huila y PNN Puracé. [...]

La organización campesina ha dado un primer paso al identificar lugares que merecen ser protegidos en virtud de su valiosa contribución a la generación de agua. Conviene dar el siguiente paso hacia un ejercicio más sistemático de registro y de inventario -si cabe la palabra- propiciado por los mismos campesinos que son quienes mejor conocen su territorio, con el fin de identificar los principales ecosistemas del territorio para definir sus estrategias de protección. El proceso de construcción del TCA permite considerar diversas opciones que se podrían explorar con mayor detenimiento. Por ejemplo, acudir a figuras que contempla la norma como las Reservas de la Sociedad Civil.” (Muñoz, p, 114, 2017)

Seguidamente, el tercer reto es acerca de fortalecer la organización campesina en los municipios del departamento del Cauca, pero:

"A diferencia del norte de Nariño, el sur del Cauca es una zona donde el ELN tiene una presencia importante ligada al cultivo de coca. Recientemente en el municipio de Mercaderes hubo una multitudinaria movilización en contra de los procesos de erradicación forzosa de coca que se han venido adelantando por parte del Ejercito nacional. Los manifestantes se oponen a un proceso de erradicación por la fuerza y piden al gobierno que se realice de manera concertada con programas serios de sustitución de cultivos y una nueva mirada [...] en el municipio de Mercaderes, fue asesinado por sicarios, Faiver Cerón Gómez, presidente de la JAC del corregimiento de Esmeraldas. El líder social había sido un recio protector de los recursos naturales de la zona; denunció y encabezó la movilización de la comunidad contra la minería ilegal en el Río Sambingo" (Muñoz, p, 116, 2017).

El ultimo reto tiene que ver con la reforma agraria integral:

"La RRI del Acuerdo final de la Habana, ratificado por el Congreso de la República, soporta su propuesta de reforma estructural del campo en el acceso progresivo y la regularización de la propiedad rural, en la que se promueve una distribución más equitativa de la tierra en cumplimiento de su función social. En esa dirección apuntan la creación del Fondo de Tierras, la formación y actualización del sistema de Catastro, los estímulos a la producción agropecuaria, entre otras disposiciones. 
Sin embargo, el texto no tiene ninguna referencia explícita a la cuestión sobre la territorialidad campesina. En cambio, la integración del campesinado aparece vía generación de espacios institucionales participativos en los que las comunidades puedan incidir en la planeación, implementación y seguimiento de los planes y programas acordados.

Las perspectivas en ese sentido para los TCA no son prometedoras. Es evidente que, para el Estado, la figura de las ZRC es suficiente reconocimiento de una territorialidad campesina. Así lo expresa cuando afirma que las ZRC son iniciativas agrarias que contribuyen a la construcción de paz, a la garantía de los derechos políticos, económicos, sociales y culturales de los campesinos y campesinas, al desarrollo con sostenibilidad socioambiental y alimentaria y a la reconciliación de colombianos y colombianas (punto 1.1.10).

Por lo demás, se compromete a apoyar formas de organización de la población rural y de la economía campesina para comunidades rurales ubicadas en áreas de manejo ambiental especial, no mucho más.

En síntesis, el espíritu de la RRI no está basado en el progresivo logro de autonomía por parte del campesinado en su territorio. Pero tampoco está completamente opuesto al mismo. Es en ese espacio de ambigüedad donde la causa por un territorio campesino tiene oportunidad. Para tal fin, las organizaciones campesinas del nivel nacional y las comunidades locales en donde se vaya a adelantar la RRI, deben hacer valer sus espacios reconocidos de participación para ampliar la visión de la RRI y ensanchar su marco de acción hacia la conformación de ciertas estructuras mínimas de gobierno del territorio” (Muñoz, p, 118, 2017).

\section{Los retos se plantean sobre un terreno minado}

El gobierno nacional también ha planteado una política territorial que busca hacer llegar la institucionalidad estatal para solucionar el conflicto social y armado. Se conoce como "Zonas Estratégicas de intervención Integral" o Zonas futuro cuyos objetivos son: "transformar los territorios llevando institucionalidad con legalidad, emprendimiento y equidad, en las regiones más afectadas por la violencia, la criminalidad y la pobreza". Continúa la explicación oficial: "[...] lograr el control institucional, garantizar los derechos ciudadanos, conseguir la disrupción de las economías ilícitas, así como preservar y defender el medio ambiente". 4

Lo anterior es la primera mina que se atraviesa en las comunidades macizeñas para buscar alternativas a los dos retos de fortalecer las organizaciones campesinas en los municipios del Cauca y en la satisfacción de las NBI. Debido a que un gobierno que reproduce la doctrina que desconoce el carácter político del conflicto

${ }^{4}$ https://id.presidencia.gov.co/Documents/190808-Infografia-Zonas-Futuro.pdf 
armado y estuvo en desacuerdo con los acuerdos de La Habana, como es el actual, no encontrará soluciones que resuelvan de raíz los conflictos sociales del Macizo; se prevé por el contrario que a través de las Zonas futuro, se le eche gasolina a las tensiones de este territorio.

Un ejemplo de esto es el caso del Catatumbo (una de las regiones incluidas en este programa) donde aumentó el pie de fuerza militar cuando inició la presidencia Duque, con la Fuerza de Tarea numero 3 (FUDRA) de cinco mil efectivos, sumándose a los trece mil efectivos calculados en el 2018. El mismo presidente Duque anunció la inversión de la fundación Buffet ${ }^{5}$ en proyectos de vías terciarias e infraestructura bajo un monto de entre 150 y 200 millones de dólares. Pero lo que sospecha el Comité de Integración Social del Catatumbo CISCA- es que esa gran inversión sea en realidad para garantizar la infraestructura requerida para la explotación de petróleo y carbón en esta región, ya que paralelo al anuncio de la inversión privada norteamericana existe dos solicitudes de explotación de carbón con los proyectos concesión Rio de oro y concesión La esmeralda; junto a la intención de reactivar pozos petroleros en los municipios de Zulia y Sardinata (CISCA, 2020). Seguido a esto las denuncias por asesinatos a líderes sociales, ex combatientes de la extinga guerrilla FARC-EP, como enfrentamientos entre insurgencias han aumentado por parte de las organizaciones campesinas y defensoras de Derechos Humanos.

Ahora, por un lado, se evidencia el incremento de las Fuerzas militares en las zonas más golpeadas por el conflicto armado, el lado económico es la otra cara del campo minado para las comunidades del país y del Macizo. En este caso, el Plan Nacional de Desarrollo 2018-2022 llama la atención en la distribución de los recursos económicos para llevarlo a cabo y también llama la atención quienes son los financiadores. Al respecto, el Senador campesino Alberto Castilla explica que la mayor inversión en el Plan Plurianual de Inversiones 2019-2022 viene del sector privado con \$363.4 billones (destacándose su inversión en educación, minería, vivienda y salud) a la par del presupuesto general de la nación que es de $\$ 352.2$ billones, de los cuales el 96\% se dirigen Defensa y Policía (Castilla, 2019).

En sintonía con lo anterior, la RRI también se encuentra lejos de ser posible gracias al panorama visto desde las políticas del actual gobierno nacional. Este análisis se ubica al exterior del TECA y de las comunidades que la sostienen, pero al interior de este actor y de la figura territorial hay otro reto que imprime debates y reflexiones sobre la proyección del TECA.

Dentro de los retos que expone Muñoz, menciona una alternativa conocida como Reservas de conservación de la sociedad civil, que según Parques Naturales Nacionales de Colombia (PNN): "son áreas protegidas privadas 
establecidas a voluntad de los propietarios de predios dedicados a la conservación de muestras de ecosistemas naturales. En estas reservas, además de la conservación, se pueden tener también sistemas de producción sostenibles, ecoturismo, educación ambiental y habitación permanente, entre otras actividades." (PNN, 2020).

Podría blindar de cierto modo los bienes de la naturaleza que están dentro del macizo, pero puede convertirse en un arma de doble filo para la organización que establezca la figura, pues está supeditada a lo que pida PNN sobre el uso de ese suelo limitando así su trabajo territorial y productivo. Como también podría haber un choque de trenes entre la Reserva de Protección de la Sociedad Civil y los mandatos del PVC y del TECA, pues el reto de la figura legal se menciona dentro de la figura territorial del TECA del macizo.

Otro choque de trenes con la Figura de Conservación de la Sociedad Civil se da con las licitaciones que se tramitan en la Agencia Nacional de Minería, sabiendo el enfoque extractivo del modelo económico impuesto en Colombia. Efectivamente en la zona del TECA del macizo se encuentran solicitudes mineras desde el 2018 y títulos mineros desde el 2014 hasta el 2018, según el Sistema de Información Geográfico, Poblacional y Estadístico -SIGPE- de la Universidad Javeriana, sede Cali (ver mapa 02).

Además, en el norte del departamento de Nariño existen cuatro (4) títulos mineros vigentes y treinta y ocho (38) permisos de exploración minera (Muñoz, 2017) .

\section{Consideraciones finales}

El actual artículo es una forma de reconocimiento de la investigación hecha por Muñoz con las comunidades campesinas del macizo y con el CIMA, ya que visibiliza estas experiencias que se realizan en esta parte del suroccidente colombiano por parte del movimiento campesino. Además de reconocer el refuerzo del mundo académico con este tipo de trabajos, porque también ayuda a fortalecer conceptos y prácticas de carácter contrahegemónicas creadas desde la base campesina.

El querer abordar los retos expuestos por el autor van en la línea argumentativa anterior, la de poder complementar el escenario político y normativo en el que se encuentran los retos del TECA del macizo para contribuir a una búsqueda de alternativas eficaces.

A pesar de tener una historia de bloqueos y retrocesos la bandera de la RRI del movimiento campesino colombiano, el TECA es una opción de facto que avanza en la dirección de la RRI en materia de gobierno propio, constituir de manera legítima un ordenamiento territorial campesino, que a través de la movilización y 
organización, las comunidades deciden qué hacer con el suelo, como convivir con los bienes comunes potenciando la agricultura sin destruir la naturaleza.

Actualmente el TECA, al igual que otras figuras territoriales de otras organizaciones campesinas colombianas, logran no solo materializar parcialmente la RRI sino también se articulan con la lucha del reconocimiento del campesinado como sujeto político de derechos.

Por otro lado, el TECA del macizo no es el único que impulsa el CNA, en otras regiones como Arauca, otras zonas del departamento del Cauca, se han constituido TECAS con diferentes avances entre sí; cada TECA se incluye en el contexto diferenciado de estos territorios poniendo sobre la mesa del CNA debates acerca de la proyección y sostenibilidad de la figura territorial campesina, debelando así lo que Fals Borda (1957) hablaba sobre la comunidad, la cual funciona como un grupo religioso, económico y recreativo con unas pautas más o menos formales y explícitas; lo que a su vez debela el tema de mayor autonomía de municipios y vecindarios para que hubiera un trabajo complementario entre campesinado y Estado en palabras de Borda (claro visto desde la visión de Reforma Agraria de Borda) (1959).

Es decir, Colombia al estar constituido por regiones y municipios, comunidades cultural y políticamente diferenciadas, es obvio que se necesita un diálogo entre estos actores y jurisdicciones bajo una estructura social coherente con esa naturaleza del país. Los TECAS son ejemplo de ello, al igual que los resguardos indígenas y títulos colectivos del pueblo afro.

\section{Referencias bibliográficas}

Castilla, Alberto (2020). Plan Nacional de Desarrollo 2018-2022. Bogotá. https://kavilando.org/images/stories/documentos/PresentacionPND_aspectos-generales.pdf

Cifuentes, Sonia (2018) Buen Vivir en Colombia, una apuesta de vida desde el campesinado Catatumbero (Tesis de maestría). Universidad Santo Tomas de Aquino. Bogotá

Coordinador Nacional Agrario (2015). Territorios Agroalimentarios. Producción, naturaleza, política y cultura campesina. Bogotá.

Coordinador Nacional Agrario (2015). Los Planes de Vida Comunitarios, para los Territorios Agroalimentarios. Secretaría de Tierras.

Dussel, Enrique (2007) Materiales para una política de la liberación. Plaza y Valdés Editores.

Dussel, Enrique (2001). Hacía una política crítica. Editorial Desclée de Brouwer, Bilbao.

Fals Borda, Orlando (1957). El Hombre y la Tierra en Boyacá. Bases históricas y sociológicas e históricas para una reforma agraria. Bogotá: Antares, documentos colombianos. 
Fals Borda, Orlando (1959). El vínculo con la tierra y su evolución en el departamento de Nariño. Revista Colombiana de Ciencias, X (41), pp.IX-XIV.

Líder social del Comité de Integración Social del Catatumbo (20 de abril de 2020). Contexto del Catatumbo. (W. Castillo, Entrevistador)

Líder social de Comité de Integración del Macizo Colombiano -CIMA- (12 de marzo de 2020)

Muñoz, Nicolás (2017) Hacia la construcción de una territorialidad campesina: La iniciativa del territorio campesino agroalimentario del norte de Nariño (Tesis de maestría). Universidad Nacional. Bogotá

Navarro, Camilo (06 de junio de 2014). Marquetalia y el inicio del conflicto armado contemporáneo. Prensa rural. http://www.prensarural.org/

Organización de las Naciones Unidas para la Alimentación y la Agricultura, Agencia Nacional de Tierras (2019). Zonas de Reserva Campesina, retos y experiencias significativas en su implementación. Bogotá.

Parques Nacionales Naturales de Colombia (2020). Reservas de la Sociedad Civil. Colombia: http://www.parquesnacionales.gov.co/portal/es/ecoturismo/reservas-de-la-sociedad-civil/ 